\title{
Research on Bidding Decision-making of International Public-Private Partnership Projects
}

\author{
Zhen Yu HU, Shui Bo ZHANG, Xin Yan LIU \\ College of Management and Economic, Tianjin University, Tianjin 300072, China
}

\begin{abstract}
In order to select the optimal quasi-bidding project for an investment enterprise, a bidding decision-making model for international PPP projects was established in this paper. Firstly, the literature frequency statistics method was adopted to screen out the bidding decision-making indexes, and accordingly the bidding decision-making index system for international PPP projects was constructed. Then, the group decision-making characteristic root method, the entropy weight method, and the optimization model based on least square method were used to set the decision-making index weights. The optimal quasi-bidding project was thus determined by calculating the consistent effect measure of each decision-making index value and the comprehensive effect measure of each quasi-bidding project. Finally, the bidding decision-making model for international PPP projects was further illustrated by a hypothetical case. This model can effectively serve as a theoretical foundation and technical support for the bidding decision-making of international PPP projects.
\end{abstract}

\section{Introduction}

PPP (Public-Private Partnership), a new type of project construction model, is widely used in infrastructure projects. And as there are more and more international projects with the participants from multiple countries, the number of PPP projects has shown a rapid growth trend in the international construction market [1]. Some investment enterprises want to bid for international PPP projects to get the opportunity to undertake them and then obtain high revenue. But compared with general projects, international PPP projects take more time and funds in the bidding, because of the massive investment and high project complexity of these international PPP projects, together with the huge difference in institutional environments. If an improper international PPP project is selected, the investment enterprise will not only suffer from a waste of resources and the miss of better project bidding opportunities, but also face enormous risks that can cause project failure in the implementation of the project [2]. Therefore, the investment enterprises that intend to invest in the international PPP projects must adopt scientific bidding decision-making methods to make rational bidding decisions in the bidding of international PPP projects.

At present, some scholars have conducted researches on bidding decision-making methods. Han [3] used a cross-impact analysis method (CIA) to develop a risk-based bidding decision-making model applied to general projects. Based on the project portfolio management theory, $\mathrm{Li}$ [4] adopted the fuzzy comprehensive evaluation method through analyzing project profitability to make bidding decisions of general projects. Liu [5] proposed a multi-objective bidding decision-making method based on the fuzzy set theory to solve the bidding decision-making problem in the bidding of general projects. However, these studies were mainly focused on general projects, and there has been relatively little scientific research on the bidding decision-making of international PPP projects. Therefore, this paper aims to establish a bidding decision-making model for international PPP projects to select the optimal project from the quasi-bidding projects.

\section{Bidding decision-making index system for international PPP projects}

Based on the characteristics of the international PPP projects, this study searched for the literature related to the bidding decision-making of international PPP projects through the Google Scholar. A total of 84 key papers were screened out and analyzed by adopting the literature frequency statistics method [6]. Then the bidding decision-making indexes which appear in at least 10 papers, were selected and used to construct the bidding decision-making index system for international PPP projects as shown in Fig 1.

*Corresponding author: Email: huzhenyugg@tju.edu.cn 


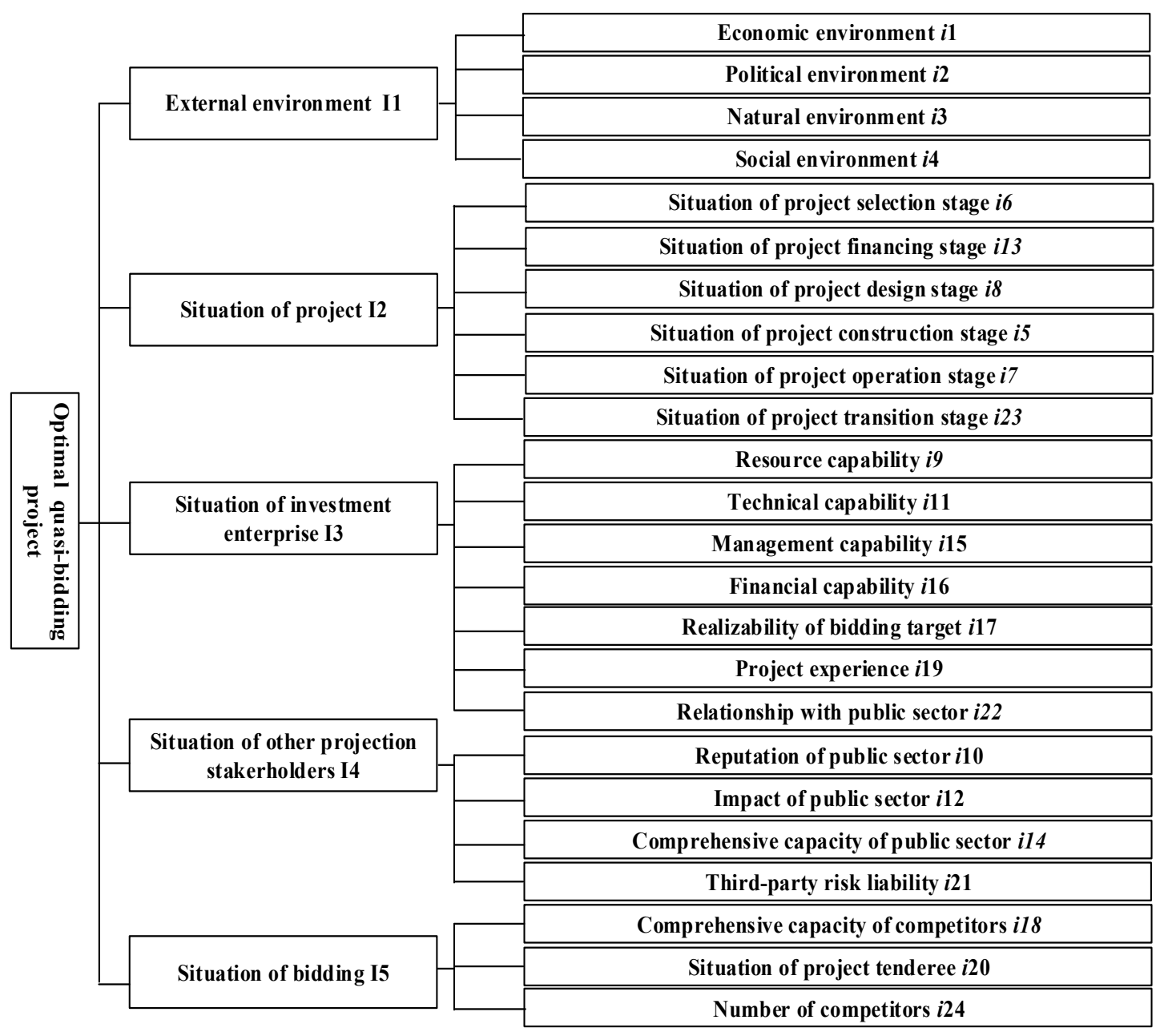

Figure 1 Bidding decision-making index system for international PPP projects

\section{Bidding decision-making model for international PPP projects}

It is assumed that the number of the quasi-bidding projects is $l$, and the number of bidding decision-making indexes is $m$. In the bidding decision-making model, $y_{i h}$ represents the index value of the project $h(h=1,2, \mathrm{~L}, l)$ under the bidding decision-making index $i(i=1,2, \mathrm{~L}, m)$, and $Y=\left(y_{i h}\right)_{m \times l}$ refers to the matrix of the decision-making index values. As the above bidding decision-making index system for international PPP projects is adopted in this study, the value of $m$ in this study equals to 24 . Among the bidding decision-making indexes in the bidding decision-making index system, only the bidding decision-making indexes of 'Number of competitors' is a quantitative bidding decision-making index, and the other 23 bidding decision-making indexes are all qualitative bidding decision-making indexes. The value of a quantitative decision-making index is determined by collecting empirical data, while the experts' evaluation scores are used to determine the value of a qualitative bidding decision-making index.

\subsection{Setting decision-making index weights}

In the proposed bidding decision-making model, the group decision-making characteristic root method [7] is used to set the subjective weights of the bidding decision-making indexes, which can be combined with the objective weights of the bidding decision-making indexes set by the entropy weight method [8] to obtain the comprehensive weights of the bidding decision-making indexes as the decision-making index weights. Setting the comprehensive weights of the bidding decision-making indexes can both make full use of the knowledge and experience of experts, and effectively make up for the shortcomings of subjective judgments, thus making the bidding decisions more scientific and reasonable.

1) Determination of subjective weights of bidding decision-making indexes through group decision-making characteristic root method

First of all, experts are invited to score the relative weights of the first-level bidding decision-making indexes under the general objective, and the relative weights of the second-level bidding decision-making indexes under their first-level bidding decision-making indexes. For example, the expert group determines the 
score matrix of $Q=\left(q_{i j}\right)_{m \times n}$ representing the relative weights of certain second-level bidding decision-making indexes under their first-level bidding decision-making index, and $q_{i j}$ refers to the relative weight of the bidding decision index $i$ judged by the expert $j(j=1,2, \mathrm{~L}, n)$. The multiplication of the scoring matrix $Q$ with its transpose is denoted as matrix $F$, that is $F=Q Q^{T}$. The expert group's judgment vector of the relative weights of the bidding decision-making indexes is a positive eigenvector corresponding to the maximum positive eigenvalue of the matrix $F$ [7]. So after obtaining and normalizing this positive eigenvector, the subjective relative weights of bidding decision-making indexes can be determined. And then according to the hierarchical structure of the bidding decision-making index system, the subjective comprehensive weights of bidding decision-making indexes represented by $\omega=\left(\omega_{1}, \omega_{2}, \Lambda, \omega_{m}\right)^{T}$ can be obtained.

2) Determination of objective weights of bidding decision-making indexes through entropy weight method

The greater the variability of a decision-making index value is, the smaller its entropy but the larger its weight is, thus the entropy weight method can be used to determine the objective weights of the bidding decision-making indexes by analyzing the degree of variation of each decision-making index value [8].

At first, the matrix $Y=\left(y_{i h}\right)_{m \times l}$ is normalized to obtain the matrix $R=\left(r_{i h}\right)_{m \times l}$. Among the 24 bidding decision-making indexes used in this study, only the bidding decision-making indexes of "Impact of public sector", "Third-party risk liability", "Comprehensive capacity of competitors" and "Number of competitors" are cost-type bidding decision-making indexes, whose values are better when they become smaller, while the remaining bidding decision-making indexes are benefit-type bidding decision-making indexes, whose values are better when they become larger.

The normalized equation for the benefit-type bidding decision-making indexes is [8]:

$$
r_{i h}=\frac{y_{i h}-\min _{1 \leq h \leq l}\left\{y_{i h}\right\}}{\max _{1 \leq h \leq l}\left\{y_{i h}\right\}-\min _{1 \leq h \leq l}\left\{y_{i h}\right\}}
$$

Similarly, the normalized equation for the cost-type bidding decision-making indexes is [8]:

$$
r_{i h}=\frac{\max _{1 \leq h \leq l}\left\{y_{i h}\right\}-y_{i h}}{\max _{1 \leq h \leq l}\left\{y_{i h}\right\}-\min _{1 \leq h \leq l}\left\{y_{i h}\right\}}
$$

The equation used to calculate the entropy of the bidding decision-making indexes is [8]:

$$
E_{i}=-k \sum_{h=1}^{l} p_{i h} \ln p_{i h}, \quad i=1,2, \Lambda, m
$$

$$
p_{i h}=\frac{r_{i h}}{\sum_{h=1}^{l} r_{i h}}\left(\text { or } r_{i h}=0 \text { with } p_{i h} \ln p_{i h}=0\right), k=\frac{1}{\ln l},
$$

\section{$0 \leq E_{i} \leq 1$}

The equation to obtain the objective weights of the bidding decision-making indexes is [8]:

$$
\begin{gathered}
\mu_{i}=\frac{1-E_{i}}{m-\sum_{i=1}^{m} E_{i}}, \quad i=1,2, \Lambda, m \\
0 \leq \mu_{i} \leq 1, \sum_{i=1}^{m} \mu_{i}=1
\end{gathered}
$$

3) Determination of comprehensive weights of bidding decision-making indexes through optimization model based on least square method

It is assumed that $w_{i}(i=1,2, \ldots, m)$ is the comprehensive weight of the bidding decision-making index $i$ and $\sum_{i=1}^{m} w_{i}=1$, so $W=\left(w_{1}, w_{2}, \Lambda, w_{m}\right)^{T}$ represents the comprehensive weight vector. To combine the subjective weights and the objective weights of the bidding decision-making indexes, the optimization model based on least square method can be expressed by the equation as [9]:

$$
\left\{\begin{array}{l}
\min I(W)=\sum_{h=1}^{l} \sum_{i=1}^{m}\left\{\left[\left(\omega_{i}-w_{i}\right) r_{i h}\right]^{2}+\left[\left(\mu_{i}-w_{i}\right) r_{i h}\right]^{2}\right\} \\
\text { s.t. } \sum_{i=1}^{m} w_{i}=1 \\
0 \leq w_{i} \leq 1, i=1,2, \Lambda, m
\end{array}\right.
$$

It is has been proved that the exact solution of this equation, i.e. the comprehensive weight, is equivalent to the arithmetic mean of the objective weight and the subjective weight, so the comprehensive weights of the bidding decision indexes can be obtained by the equation expressed as [9]:

$$
w_{i}=\frac{\omega_{i}+\mu_{i}}{2}, \quad i=1,2, \Lambda, m
$$

\subsection{Evaluating quasi-bidding projects}

1) Transformation of decision-making index values into consistent effect measure

Because of the various dimensions and heterogeneous nature of the decision-making index values, consistent effect measure function can be constructed to transform the decision-making index value $y_{i h}$ into consistent effect measure $d_{h}^{(i)} \in[-1,1](h=1,2, \mathrm{~L}, l ; i=1,2, \mathrm{~L}, m)$, to make these decision-making index values comparable. The consistent effect measure matrix $D^{(i)}=\left(d_{h}^{(i)}\right)$ can be 
obtained accordingly [10].

At first, the critical value $y_{i h_{0}}$ of the bidding decision-making index $i$ is determined by averaging the critical values of this bidding decision-making index set by each expert. Then the consistent effect measure is calculated based on the following two equations. The larger the value of $d_{h}^{(i)}$ is, the more satisfactory the decision-making index value $y_{i h}$ is. ' $d_{h}^{(i)} \in[0,1]$ ' means the decision-making index value of the project $h$ under the decision-making index $i$ meets the requirements, but ' $d_{h}^{(i)} \in[-1,0)$ ' has the opposite meaning.

If the satisfactory range of a benefit-type decision-making index value is $y_{i h} \in\left[y_{i h_{0}}, \max y_{i h}\right]$,

the consistent effect measure function for the benefit-type decision-making indexes can be proposed as [10]:

$$
d_{h}^{(i)}=\frac{y_{i h}-y_{i h_{0}}}{\max y_{i h}-y_{i h_{0}}}
$$

Similarly, if the satisfactory range of a cost-type decision-making index value is $y_{i h} \in\left[\min y_{i h}, y_{i h_{0}}\right]$, the consistent effect measure function for the cost-type decision-making indexes can be proposed as [10]:

$d_{h}^{(i)}=\frac{y_{i h_{0}}-y_{i h}}{y_{i h_{0}}-\min y_{i h}}$

2) Calculation of comprehensive effect measure of quasi-bidding projects

According to the following equation, the comprehensive effect measure $d_{h} \in[-1,1]$ of the quasi-bidding project $h$ can be calculated by synthesizing the weights of each bidding decision-making index [10]. The larger the value of $d_{h}$ is, the more satisfactory the quasi-bidding project $h$ is. ' $d_{h} \in[0,1]$ ' means the quasi-bidding project $h$ meets the requirements of the investment enterprise, but ' $d_{h} \in[-1,0)$ ' means oppositely. Then the comprehensive effect measure matrix $D=\left(d_{h}\right)$ can be obtained, based on which the optimal quasi-bidding project can be chosen from the satisfactory quasi-bidding projects by comparing the comprehensive effect measure.

$d_{h}=\sum_{i=1}^{m} w_{i} \bullet d_{h}^{(i)}$

If ' $\max _{1 \leq h \leq l}\left\{d_{h}\right\}=d_{h_{0}}$ ' exists, the quasi-bidding project $h_{0}$ is the optimal quasi-bidding project.

\section{Hypothetical case}

An investment enterprise intends to bid for an international PPP project which is chosen from Project $h_{1}$, Project $h_{2}$ or Project $h_{3}$, and a expert group with five members is set up to analyze the bidding decision to select the optimal quasi-bidding project.

\subsection{Determination of decision-making index values}

The expert group uses the Likert five-component method to score the 23 qualitative decision-making index values of each quasi-bidding project. The score increases from 1 to 5 , indicating the gradual increment of the qualitative decision-making index value. The scores of the five experts are averaged to be used as the qualitative decision-making index values. As for the only quantitative bidding decision-making index "Number of competitors", it is found there are $8,12,10$ quasi bidders in addition to this investment enterprise for Project $h_{1}$, Project $h_{2}$ and Project $h_{3}$ respectively through a survey. Therefore, all decision-making index values of the three quasi-bidding projects are obtained and shown in Table 1 .

Table 1 Decision-making index values and weights

\begin{tabular}{c|ccc|ccc}
\hline \multirow{2}{*}{ Indexes } & \multicolumn{3}{|c|}{ Decision-making index values } & \multicolumn{2}{c}{ Decision-making index weights } \\
\cline { 2 - 6 } & Project $h_{1}$ & Project $h_{2}$ & Project $h_{3}$ & $\begin{array}{c}\text { Subjective } \\
\text { comprehensive } \\
\text { weights }\end{array}$ & $\begin{array}{c}\text { Objective } \\
\text { weights }\end{array}$ & $\begin{array}{c}\text { Comprehensive } \\
\text { weights }\end{array}$ \\
\hline $\boldsymbol{i} 1$ & 3.2 & 3.8 & 3.4 & 0.067 & 0.041 & 0.05 \\
i2 & 3.4 & 4 & 3.6 & 0.064 & 0.041 & 0.05 \\
$i 3$ & 4.2 & 3.2 & 3 & 0.061 & 0.052 & 0.06 \\
$i 4$ & 4 & 3.6 & 3.4 & 0.058 & 0.041 & 0.05 \\
$i 5$ & 3.2 & 3.2 & 4.2 & 0.048 & 0.031 & 0.04 \\
$i 6$ & 3.8 & 3.2 & 3.8 & 0.042 & 0.033 & 0.04 \\
$i 7$ & 3 & 3.8 & 3.4 & 0.050 & 0.031 & 0.04 \\
i8 & 3.4 & & & 0.038 & 0.031 & 0.03
\end{tabular}




\begin{tabular}{|c|c|c|c|c|c|c|}
\hline$i 9$ & 3.8 & 4.2 & 3.8 & 0.034 & 0.083 & 0.06 \\
\hline$i 10$ & 3.8 & 3.8 & 4.4 & 0.043 & 0.083 & 0.06 \\
\hline$i 11$ & 3.2 & 3.8 & 4.2 & 0.034 & 0.033 & 0.03 \\
\hline$i 12$ & 3.2 & 4.2 & 3.4 & 0.042 & 0.031 & 0.04 \\
\hline$i 13$ & 3 & 3 & 3.6 & 0.035 & 0.083 & 0.06 \\
\hline$i 14$ & 3.6 & 3 & 3.6 & 0.041 & 0.031 & 0.04 \\
\hline$i 15$ & 3.4 & 3.2 & 4 & 0.031 & 0.045 & 0.04 \\
\hline$i 16$ & 4.2 & 4 & 2.6 & 0.039 & 0.031 & 0.03 \\
\hline$i 17$ & 3.4 & 3.2 & 3 & 0.034 & 0.035 & 0.03 \\
\hline$i 18$ & 3.2 & 3.8 & 3 & 0.041 & 0.031 & 0.04 \\
\hline$i 19$ & 3.2 & 3.6 & 3.8 & 0.031 & 0.032 & 0.03 \\
\hline$i 20$ & 4.2 & 3 & 3.8 & 0.042 & 0.032 & 0.04 \\
\hline$i 21$ & 4.2 & 3.4 & 4.4 & 0.032 & 0.049 & 0.04 \\
\hline$i 22$ & 3 & 3.2 & 3.2 & 0.029 & 0.031 & 0.03 \\
\hline$i 23$ & 3.8 & 3.6 & 3.4 & 0.025 & 0.035 & 0.03 \\
\hline$i 24$ & 8 & 12 & 10 & 0.036 & 0.035 & 0.04 \\
\hline
\end{tabular}

\subsection{Determination of decision-making index weights}

The expert group also adopts the Likert five-component method to score the relative weights of the bidding decision-making indexes, and the score ranges from 1 to 5 representing the continuum from the end of the minimum relative weight to the end of the maximum relative weight. For example, the expert group determines the score matrix of the subjective relative weights of the first-level bidding decision-making indexes under the general objective, which is

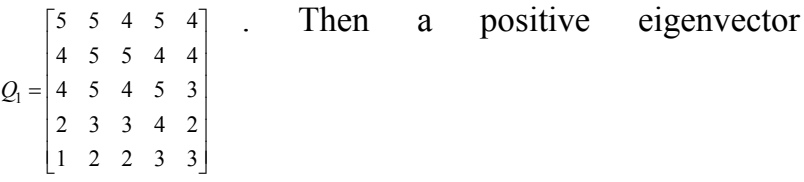

corresponding to the maximum positive eigenvalue of this matrix is obtained by using MATLAB 7.0, which is $(0.542,0.518,0.504,0.342,0.259)^{T}$. This positive eigenvector is normalized and the subjective relative weight vector of the first-level bidding decision-making indexes under the general objective is obtained, which is $\omega_{1}^{\prime}=(0.250,0.239,0.233,0.158,0.120)^{T}$. Similarly, according to the experts' score matrixes of the subjective relative weights of the second-level bidding decision-making indexes under their first-level bidding decision-making indexes, which are $\left[\begin{array}{ccccc}5 & 5 & 4 & 4 & 5\end{array}\right]$,

$$
Q_{2}=\left[\begin{array}{lllll}
5 & 5 & 4 & 4 & 5 \\
5 & 4 & 4 & 5 & 4 \\
4 & 5 & 4 & 4 & 4 \\
4 & 4 & 5 & 4 & 3
\end{array}\right]
$$

$Q_{3}=\left[\begin{array}{lllll}4 & 5 & 3 & 4 & 4 \\ 3 & 3 & 4 & 4 & 3 \\ 4 & 4 & 3 & 4 & 3 \\ 5 & 4 & 5 & 5 & 4 \\ 5 & 5 & 5 & 4 & 5 \\ 2 & 3 & 2 & 3 & 2\end{array}\right] \quad, \quad Q_{4}=\left[\begin{array}{lllll}4 & 5 & 3 & 4 & 4 \\ 4 & 3 & 4 & 4 & 5 \\ 3 & 4 & 5 & 3 & 3 \\ 5 & 5 & 4 & 5 & 4 \\ 4 & 3 & 4 & 5 & 4 \\ 3 & 4 & 3 & 4 & 4 \\ 3 & 3 & 4 & 4 & 3\end{array}\right]$,

$Q_{5}=\left[\begin{array}{lllll}4 & 5 & 5 & 5 & 4 \\ 4 & 4 & 5 & 4 & 5 \\ 4 & 5 & 4 & 4 & 5 \\ 3 & 4 & 3 & 3 & 4\end{array}\right], \quad Q_{6}=\left[\begin{array}{lllll}5 & 4 & 5 & 4 & 4 \\ 5 & 5 & 3 & 4 & 5 \\ 4 & 4 & 4 & 3 & 4\end{array}\right], \quad$ the

subjective relative weight vectors of the second-level bidding decision-making indexes under their first-level bidding decision-making indexes are obtained by calculating and normalizing with MATLAB 7.0, which are

$$
\omega_{2}{ }^{\prime}=(0.268,0.256,0.243,0.233)^{T}
$$

$\omega_{3}{ }^{\prime}=(0.177,0.148,0.159,0.201,0.209,0.106)^{T}$

$\omega_{4}{ }^{\prime}=(0.147,0.147,0.132,0.169,0.148,0.132,0.125)^{T}$

$\omega_{5}{ }^{\prime}=(0.275,0.263,0.260,0.202)^{T}$

$\omega_{6}{ }^{\prime}=(0.345,0.354,0.301)^{T}$. And then according to the hierarchical structure of the decision-making index system, the subjective comprehensive weights of the bidding decision-making indexes are obtained and shown in Table 1.

Furthermore, the decision-making index values are normalized according to the equation (1), equation (2) and equation (3). Then the objective weights of bidding decision indexes are calculated according to the equation (4) and shown in Table 1. Finally, according to the equation (6), the comprehensive weights of the bidding decision-making indexes are obtained and also shown in Table 1.

\subsection{Determination of optimal quasi-bidding}




\section{project}

The critical values of the bidding decision-making indexes are determined by averaging the critical values of these bidding decision-making indexes judged by each expert, and the vector of these critical values is obtained as $Y_{0}=[3,3,3,3,2.5,3,3,3,3,3,3,4.5,3,3,3,3,3,4,3,3,4.5,3,3,10]^{T}$, and the consistent effect measure of each bidding decision-making index satisfies the condition of $d_{h}^{(i)} \in[-1,1]$. After the consistent effect measure matrix $D^{(i)}$ is obtained according to the equation (7) and equation (8), the comprehensive effect measure matrix is obtained according to the equation (9), that is $D=W \bullet D^{(i)}=[0.64,0.51,0.59]$.

The result shows $d_{1}>0, d_{2}>0, d_{3}>0$, so the three quasi-bidding projects all meet the requirements of the investment enterprise. But $\max _{1 \leq h \leq 3}\left\{d_{h}\right\}=d_{1}=0.64$, so the Project $h_{1}$ is the optimal quasi-bidding project for the investment enterprise.

\section{Conclusion}

Through the above case analysis, the feasibility and applicability of the proposed bidding decision-making model for international PPP projects have been verified. Although the model can be applied to the bidding decision-making of international PPP projects, some suggestions are still put forward to strengthen its validity further.

Firstly, the developed model has integrated sufficient macro-level factors of the bidding decision-making of international PPP projects, such as the 'Economic environment' and 'Political environment', but some micro-level psychological factors that may affect the decision behavior of the decision maker, such as the decision maker's risk preference, were not taken into account. Future research can further improve the model by resolving the issue to make it more practical and effective.

Secondly, only a hypothetical case was used in this study to verify the proposed bidding decision-making model for international PPP projects. Future research can conduct empirical studies to collect real-life project data to further validate this model, and to provide practical proof of the conclusion of this study. Such rich empirical investigation would contribute to the further improvement of the bidding decision-making model for international PPP projects.

\section{Acknowledgements}

We gratefully acknowledge the National Natural Science Foundation of China (NSFC) for its financial support for this research work (Grant Nos. 71572124).

\section{References}

1. Grimsey D, Lewis MK. Evaluating the risks of public private partnerships for infrastructure projects[J]. International Journal of Project Management, 2002, 20(2): 107-118.

2. Zhang XQ. Critical success factors for Public-Private Partnerships in infrastructure development[J]. Journal of Construction Engineering and Management, 2005, 131(1): 3-14.

3. Han SH, Diekmann J. Making a risk-based bid decision for overseas construction projects[J]. Construction Management and Economics, 2001, 19(8): 765-776.

4. Li Hong-bing, Li Hong, Chen Yu. Application of Portfolio Management to Bidding Decision-making[J]. Journal of Wuhan University of Technology, 2008, 30(6): 170-173.

5. Liu Er-lie, Wang Jian, Luo Gang. Project bidding decision based on fuzzy logic [J]. China Civil Engineering Journal, 2003, 36(3): 57-63.

6. Lindsay $\mathrm{R} \mathrm{K}$, Gordon $\mathrm{M}$ D. Literature-based discovery by lexical statistics[J]. Journal of the Association for Information Science and Technology, 1999, 50(7): 574-587.

7. Chen Wei, Kang Xin, Feng Zhi-jun, Tian Shi-hai. Identification of evaluation indexes for intellectual property's exploitation of high-tech enterprises based on group decision-making characteristic root method[J]. Science \& Technology Progress and Policy, 2011, 28(11): 116-119.

8. Hong Wei-min, Liu Hong-mei, Wang Zhuo-fu. Project transaction mode decision based on entropy weight fuzzy comprehensive evaluation method[J]. Science and Technology Management Research, 2010(3): 122-125.

9. Xu Ze-shui. Improvement and Extension of a Decision Making Method[J]. Operations Research and Management Science, 2000, 9(4): 46-51.

10. Liu Si-feng, Yuan Wen-feng, Sheng Ke-qin. Multi-attribute intelligent grey target decision model[J]. Control and Decision, 2010, 25(8): 1159-1163. 